\title{
A Three-Dimensional Carbon Framework Constructed by N/S Co-doped Graphene Nanosheets with Expanded Interlayer Spacing Facilitates Potassium Ion Storage
}

\author{
Wenxiu Yangł, Jinhui Zhoù, Shuo Wang, Zichen Wang, Fan Lv, Wenshu Zhang, Weiyu Zhang, Qiang Sun, and \\ Shaojun Guo* \\ Department of Materials Science \& Engineering, College of Engineering, Peking University, Beijing, 100871, \\ China. \\ E-mail: guosj@pku.edu.cn \\ ${ }^{[\dagger}$ These authors contributed equally to this work
}

\section{Chemicals}

Melamine and cyanuric acid were purchased from Sigma-Aldrich. Sublimed sulphur powder (S) and Pluronic ${ }^{\circledR}$ F-127 (F127) were obtained from Aladdin. All chemicals were analytical grade and used as received. All aqueous solutions were prepared with ultrapure water from a Water Purifier System (Sichuan Water Purifier Co. Ltd., China).

\section{Material synthesis}

\section{Synthesis of 3D carbon framework material assembled by N-codoped graphene nanosheets (CFM-NG) and CFM-SnNG}

Firstly, $0.30 \mathrm{~g}$ of F127 powder was dissolved into $30 \mathrm{~mL}$ of ultrapure water by stirring for $0.5 \mathrm{~h}$. Then, $1.0 \mathrm{~g}$ of melamine and $1.0 \mathrm{~g}$ of cyanuric acid were seperately added into the F127 aqueous solution and further continuously stirred for $12 \mathrm{~h}$. The obtained milky dispersion liquid was frozen with liquid $\mathrm{N}_{2}$. Thereafter, the frozen samples were heated at $110{ }^{\circ} \mathrm{C}$ for $6 \mathrm{~h}$ in a drying oven. The remaining white product was grinded for $20 \mathrm{~min}$ in the agate mortar. After that, the product was heated stepwise at 300 and $\mathrm{T}{ }^{\circ} \mathrm{C}$ for $1 \mathrm{~h}$ and $2 \mathrm{~h}$, respectively, with a heating rate of $5{ }^{\circ} \mathrm{C} / \mathrm{min}$ in the Ar atmosphere, followed by cooling down to room temperature. For simplicity, the resulting samples were named as CFM-NG-T (T=700, 800 and $\left.900^{\circ} \mathrm{C}\right)$.

Further heatment procedure $\left(500{ }^{\circ} \mathrm{C}(1 \mathrm{~h}), 2{ }^{\circ} \mathrm{C} / \mathrm{min}\right.$ and $\left.\mathrm{Ar}\right)$ was performed with $\mathrm{X} \mathrm{mg}(\mathrm{X}=480,960$ and $1600 \mathrm{mg}$ ) $\mathrm{S}$ powder and $16 \mathrm{mg}$ CFM-NG placing on the left and right ends of the quartz boat, respectively. After that, we cooled down the system to room temperature. We named the samples as CFM- $S_{n} N G(n=X / 16=30,60,100$ denotes the mass ratio of $S$ powder and CFM-NG precusors).

\section{Characterization}

Transmission electron microscopy (TEM) measurements were conducted on a JEM-2010 microscope operated at $200 \mathrm{kV}$ and equipped with an energy-dispersive X-ray spectrometer (EDS, Bruker Quantax). The morphologies of the resulting samples were characterized by field-emission scanning electron microscopy (FESEM, Hitachi SU8010). Scanning transmission electron microscopy (STEM) analyses were carried out using a Hitachi HD2700C $(200 \mathrm{kV})$. The structure of materials was checked by X-ray diffraction measurement (XRD, Rigaku, D/mas-RB) and Raman spectra (iHR550). The element content was analyzed by X-ray photoelectron spectroscopy (XPS, Kratos XSAM 800, $\mathrm{Cu}-\mathrm{K} \alpha$ radiation). The specific surface areas and pore distribution of the resulting materials were measured according to the Brunauer-Emmett-Teller (BET) method.

\section{Electrochemical Characterization}


To measure the electrochemical performances, the CR2032 coin type half-cells were assembled in an Ar-filled glove box with both moisture and oxygen concentrations below $0.1 \mathrm{ppm}$. The mixture including $80 \mathrm{wt} \%$ of the active materials (CFM-NG and CFM-S $\mathrm{nG}$ ), $10 \mathrm{wt} \%$ acetylene black and $10 \mathrm{wt} \%$ polyvinylidene fluoride (PVDF) were dispersed in $\mathrm{N}$-metryl-2-pyrrolidone to make uniform slurries. Then, the slurries were coated onto a copper foil and dried at $80{ }^{\circ} \mathrm{C}$ for $12 \mathrm{~h}$ under vacuum. Finally, the working electrode slices were obtained by a tablet machine.

In the CR2032 coin type half-cells system, the potassium metal was regarded as the reference electrode and counter electrode, respectively, 1.0 M KFSI in a mixture of ethylene carbonate (EC) and diethyl carbonate (DEC) (1:1, v/v) was used as the electrolyte, and the glass fiber (GF/D) from Whatman was used as the separator.

Before the ex-situ test, the electrode was washed by diethyl carbonate (DEC) for more than 5 times and dried in the argon-filled glove-box before the XRD, Raman and TEM measurements. LAND-CT2001A battery-testing instrument was used to test the galvanostatic charge and discharge process at ambient temperature under different current densities, within the potential range of 0.01-2.5 V (vs. K/K ${ }^{+}$). Cyclic voltammetry (CV) measurements were recorded on Biologic VSP-300 at scanning rate of $0.1,0.2,0.5,0.8,1.0,1.2 \mathrm{mV} / \mathrm{s}$ within the same potential range.

\section{DFT calculation}

The Vienna $\mathrm{Ab}$ initio Simulation Package $^{1}$ (VASP) within projector augmented-wave ${ }^{2}$ (PAW) approach are used to perform all the density functional theory (DFT) simulations. Perdew-Burke-Ernzerhof (PBE) exchange-correction functional of the Generalized gradient approximation (GGA) is used for all structural optimization and static calculation. ${ }^{3-4}$ The energy cutoff for the plane wave is $520 \mathrm{eV}$ for pseudopotentials of $\mathrm{K}\left(4 \mathrm{~s}^{1}\right), \mathrm{C}\left(2 \mathrm{~s}^{2} 2 \mathrm{p}^{2}\right), \mathrm{N}\left(2 \mathrm{~s}^{2} 2 \mathrm{p}^{3}\right)$ and $\mathrm{N}\left(3 \mathrm{~s}^{2} 3 \mathrm{p}^{4}\right)$, the energy and force convergence criteria are $1 \times 10^{-4} \mathrm{eV}$ and $2.0 \times 10^{-2} \mathrm{eV} / \AA$, respectively. Van der Waals correction are including with DFT-D2 method of Grimme in all simulations. ${ }^{5}$

The adsorption energies per $\mathrm{K}$ atom $\left(E_{a d s}\right)$ are obtained by the following equation:

$\mathrm{E}_{\text {ads }}=\left[\mathrm{E}_{\text {total }}-\mathrm{E}_{\text {slab }}-N \mu_{K-b u l k}\right] / N$

where Etotal, Eslab and $\mu \mathrm{K}$-bulk are the energies of total electronic energies of adsorbed carbon, a bare slab without $\mathrm{K}$, and chemical potential of bulk $\mathrm{K}$. The $\mathrm{N}$ represent the change of $\mathrm{K}$ atoms during the adsorption. Supercells with a,b lattice larger than $14 \AA$ are used, and $\Gamma$-centered k-point sampling grid of $1 \times 1 \times 4$ is adopted here.

Figures

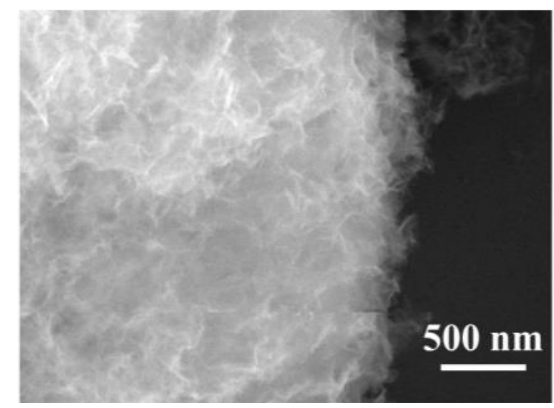

Figure S1 SEM images of CFM-S $30 \mathrm{NG}$. 


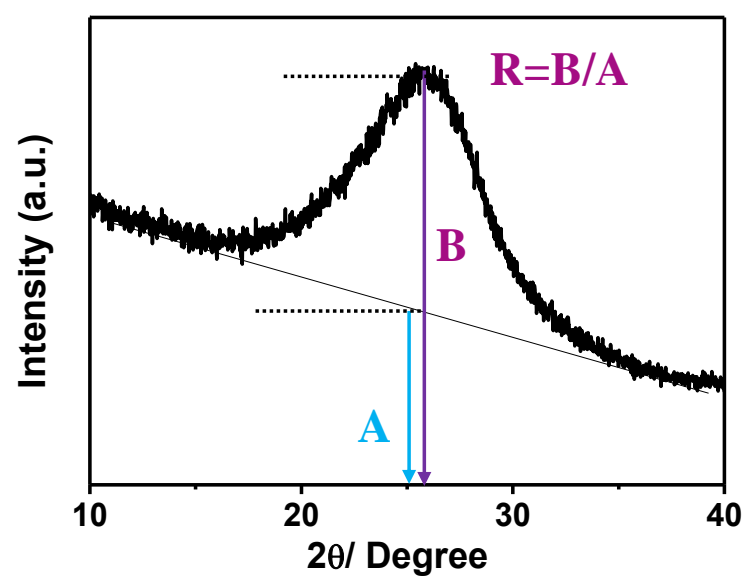

Figure S2 The R-value of CFM-NG-800 from (002) peak is equal to the intensity (B) divided by the background (A).

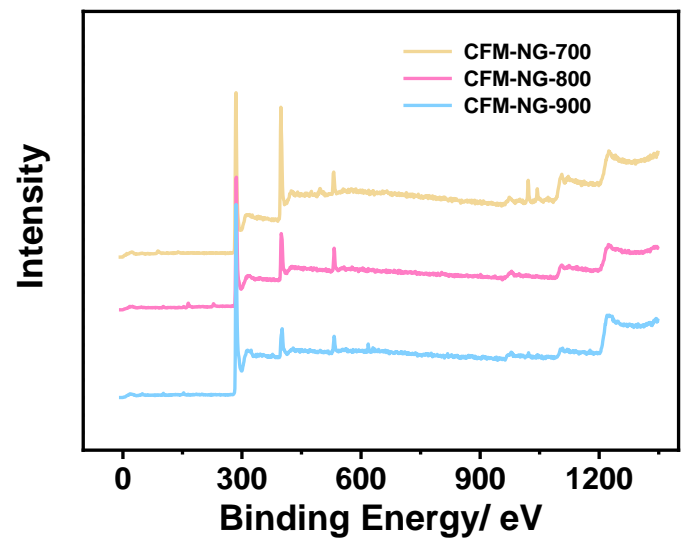

Figure S3 X-ray photoelectron spectroscopy of CFM-NG-700, CFM-NG-800 and CFM-NG-900.
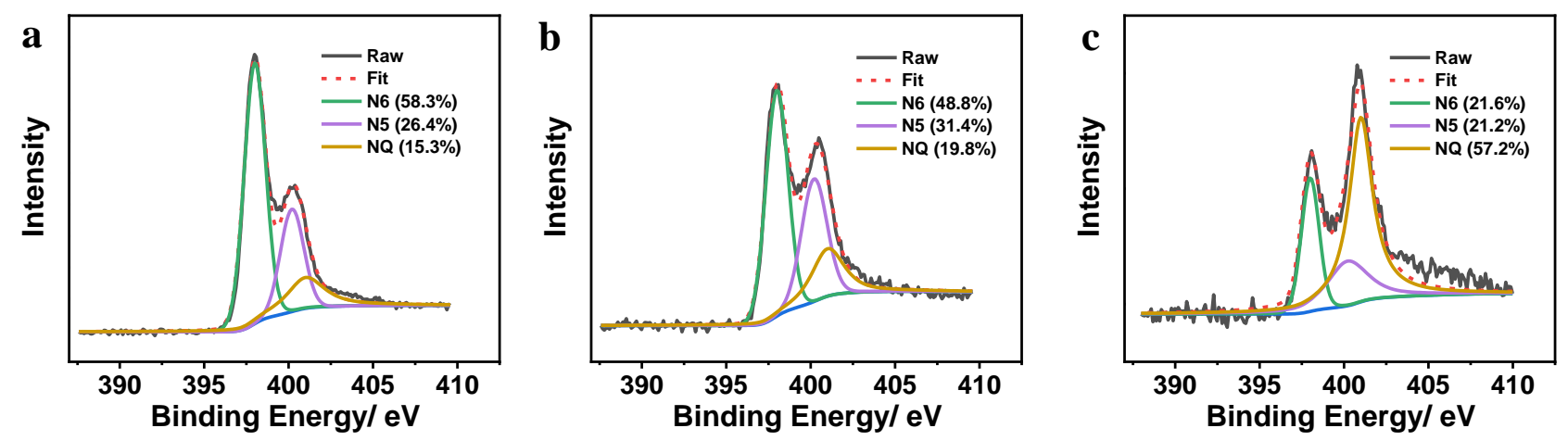

Figure S4 The high resolution N 1s XPS spectroscopy of (a) CFM-NG-700, (b) CFM-NG-800 and (c) CFM-NG-900. 


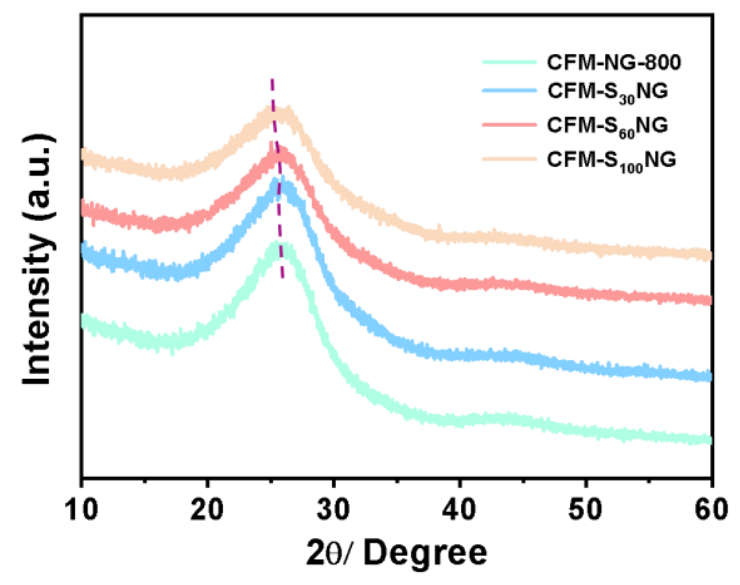

Figure S5 XRD of CFM-NG-800, CFM-S ${ }_{30} \mathrm{NG}, \mathrm{CFM}-\mathrm{S}_{60} \mathrm{NG}$ and CFM-S ${ }_{100} \mathrm{NG}$.
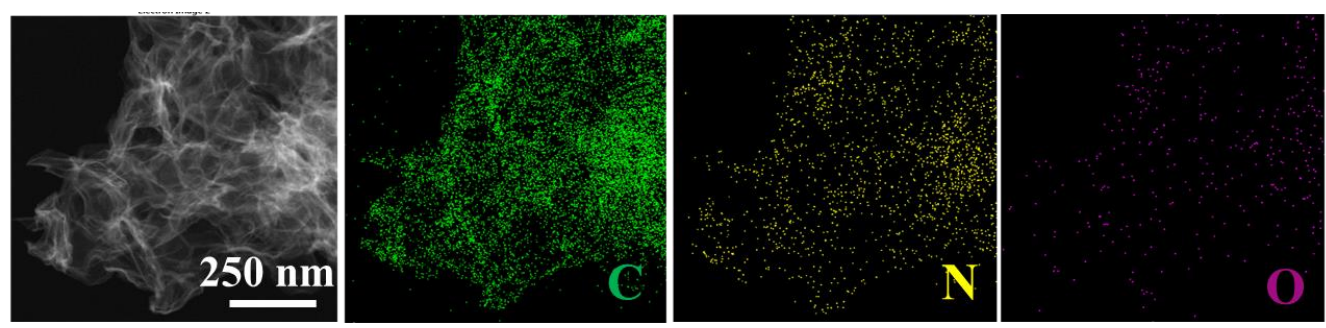

Figure S6 HAADF-STEM images and the corresponding EDS mapping of the CFM-NG-800 material.

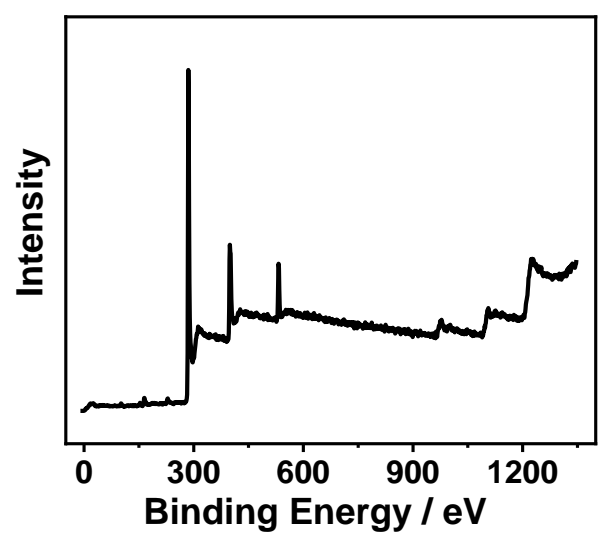

Figure S7 XPS analysis of the CFM-S ${ }_{30} \mathrm{NG}$ material. 


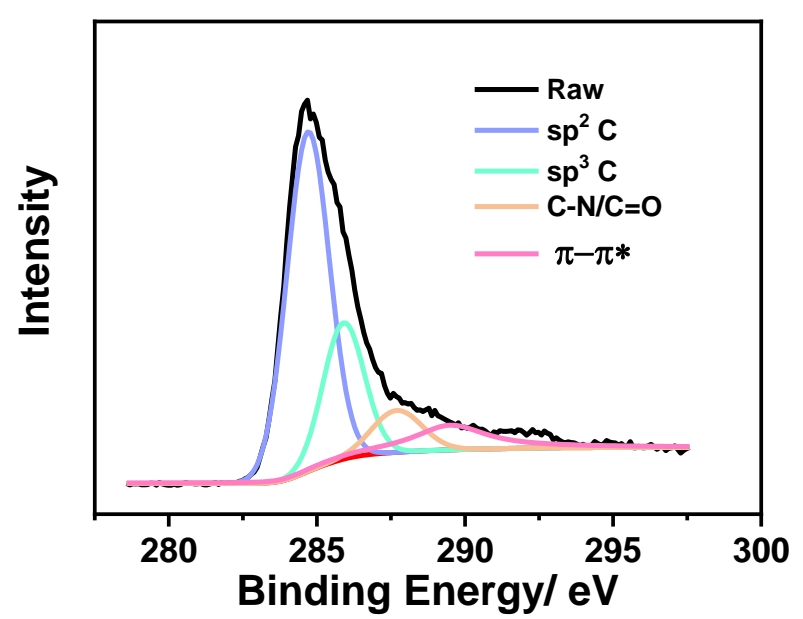

Figure S8 The high resolution C 1s XPS spectroscopy of CFM-NG-800.

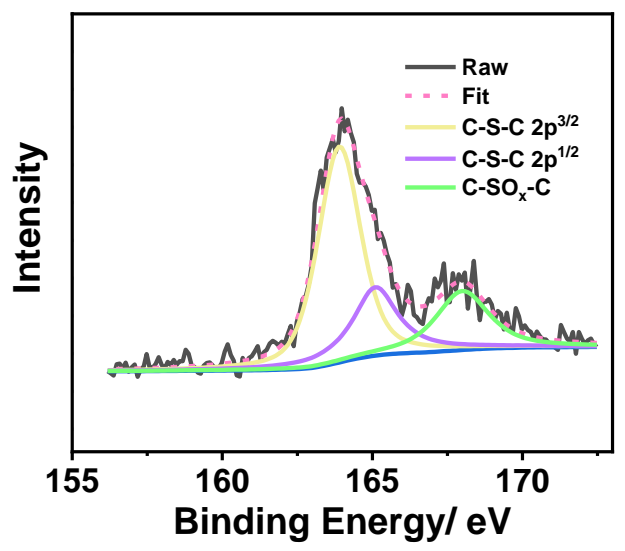

Figure S9 The high resolution S 2p XPS spectroscopy of CFM-S ${ }_{30} \mathrm{NG}$.
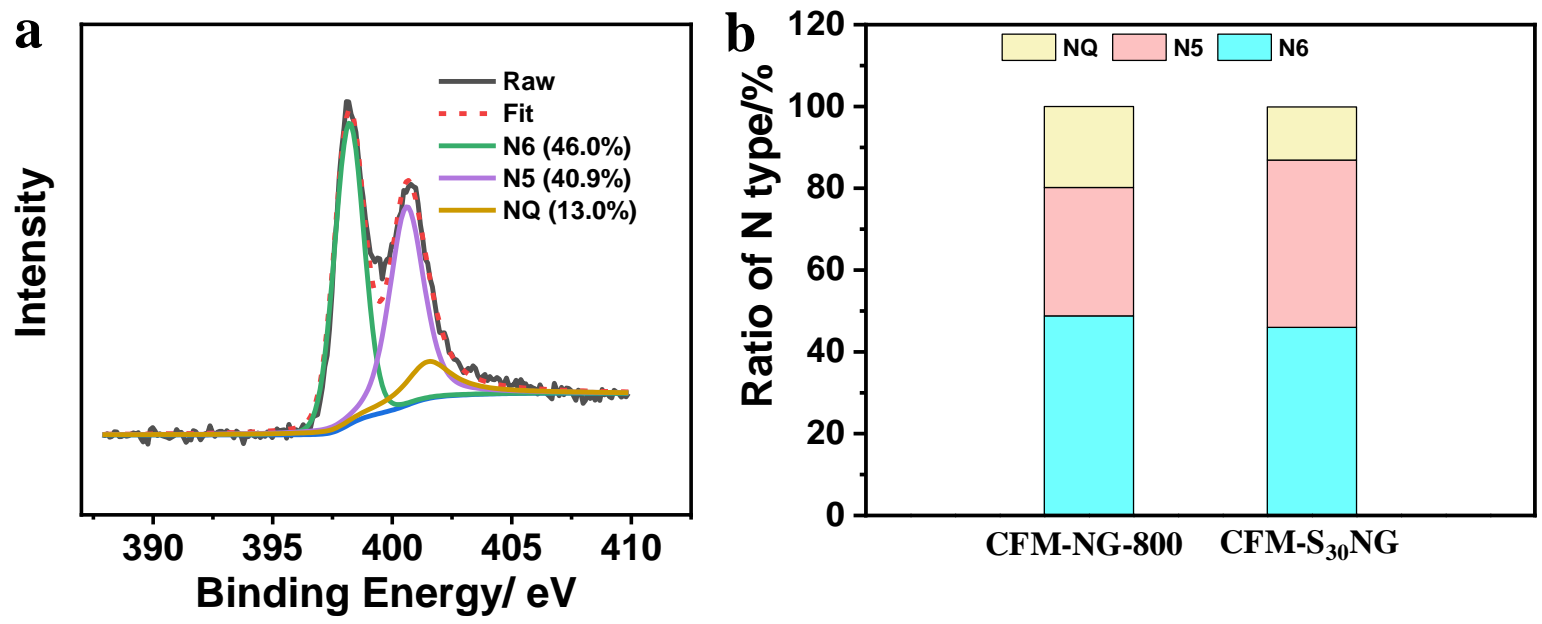

Figure S10 (a)The high resolution N 1s XPS spectroscopy of CFM-S $30 \mathrm{NG}$ and (b) Distribution of different types of $\mathrm{N}$ in the CFM-NG-800 and CFM-S $30 \mathrm{NG}$ materials. 

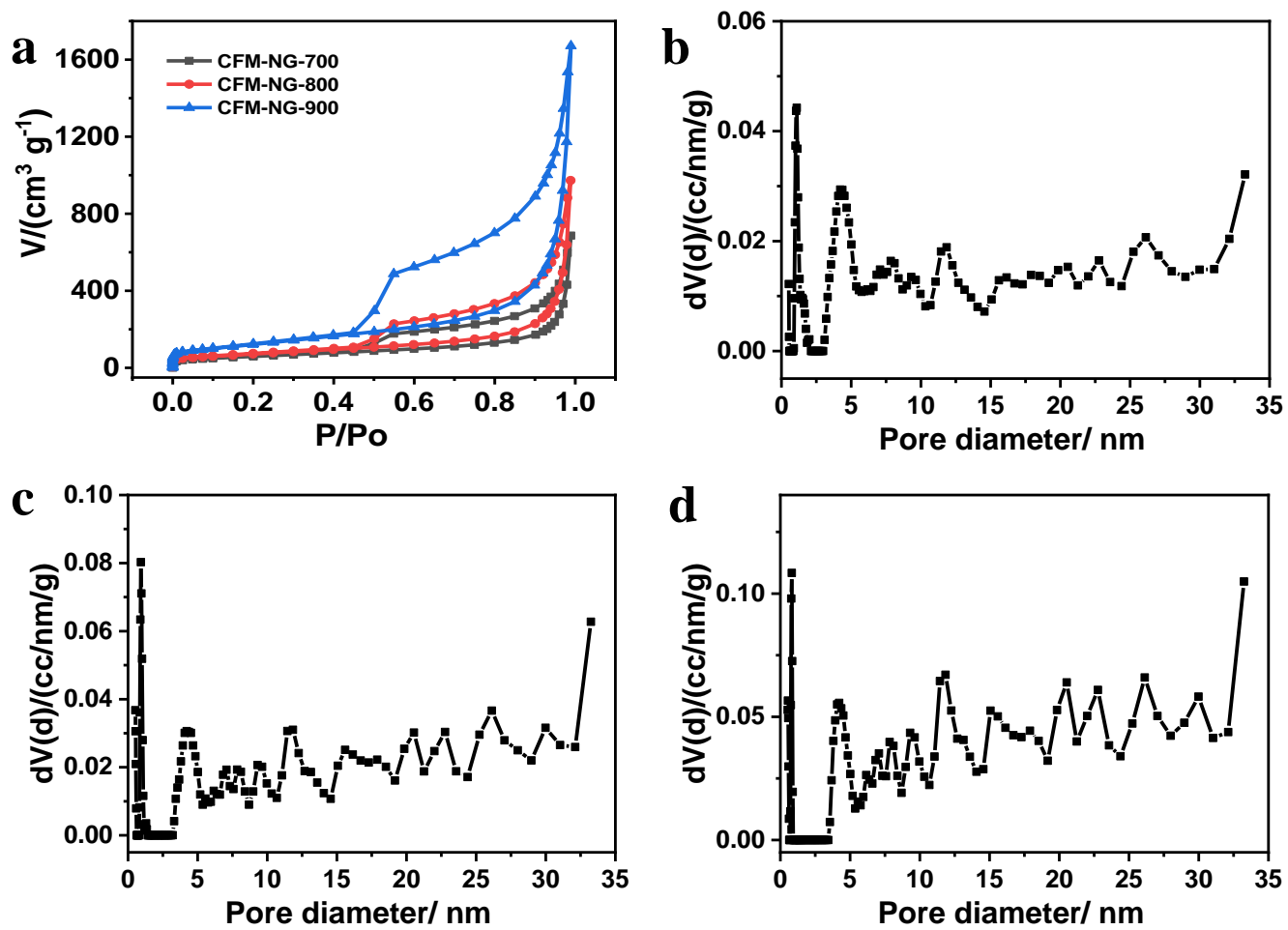

Figure S11 (a) Nitrogen adsorption-desorption isotherm curves of CFM-NG-700, CFM-NG-800 and CFM-NG-900. Pore size distributions of (b) CFM-NG-700, (c) CFM-NG-800 and (d) CFM-NG-900.

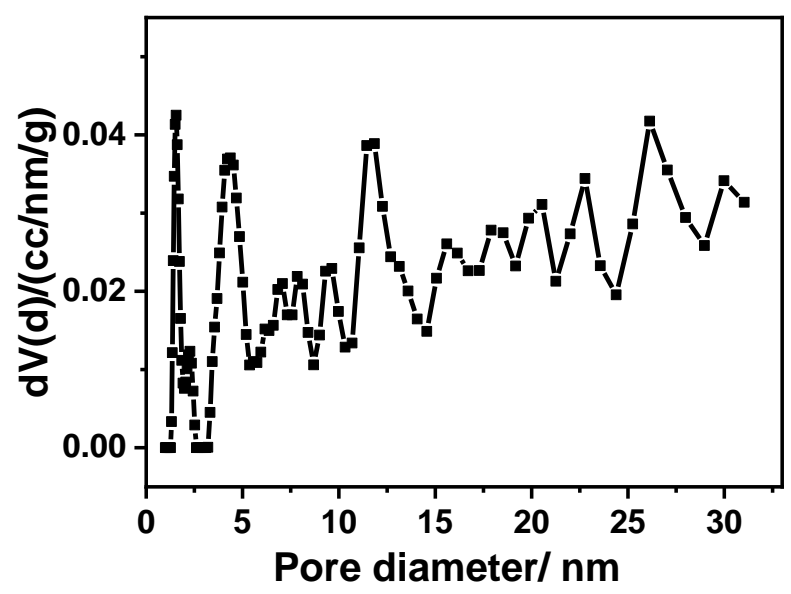

Figure S12 Pore size distribution of the CFM-S ${ }_{30} \mathrm{NG}$ material. 

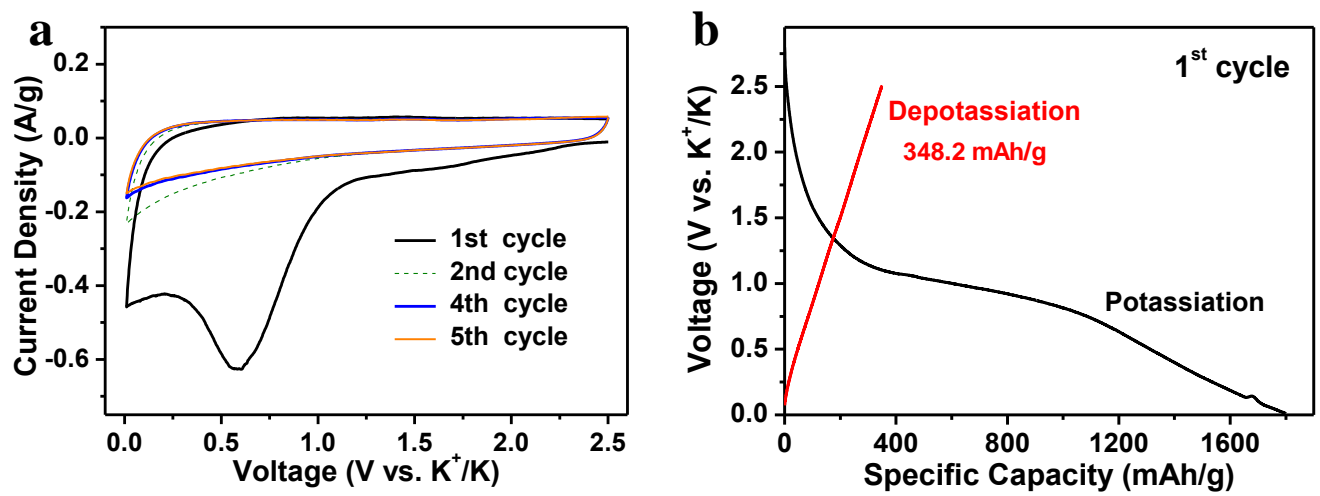

Figure S13. (a) Cyclic voltammetry (CV) curves of the $\mathrm{S}_{30} \mathrm{~N}-\mathrm{HCN}$ s electrode for PIBs between 0.01 $\mathrm{V}$ and $2.5 \mathrm{~V}$ with a scan rate of $0.1 \mathrm{mV} / \mathrm{s}$. (b) Potassiation and depotassiation profile of the CFM-S ${ }_{30} \mathrm{NG}$ electrode for the first cycle at $50 \mathrm{~mA} / \mathrm{g}$.

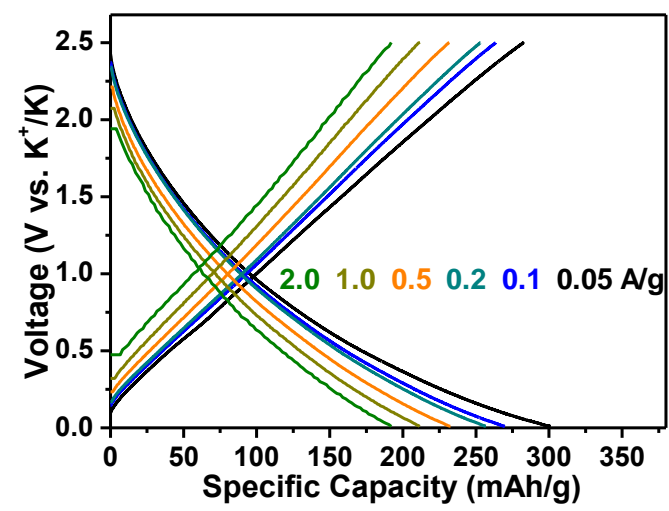

Figure S14. The potassiation and depotassiation profiles of the CFM-S ${ }_{30} \mathrm{NG}$ electrode at different current densities from 50 to $2000 \mathrm{~mA} / \mathrm{g}$.

a

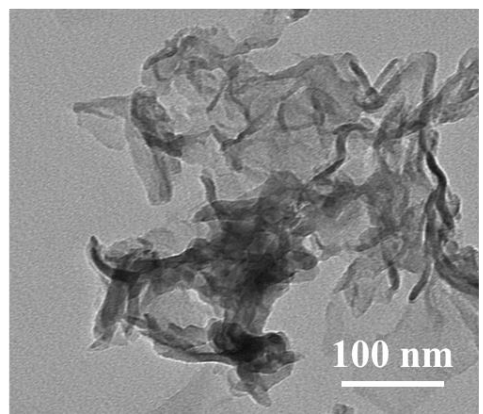

b

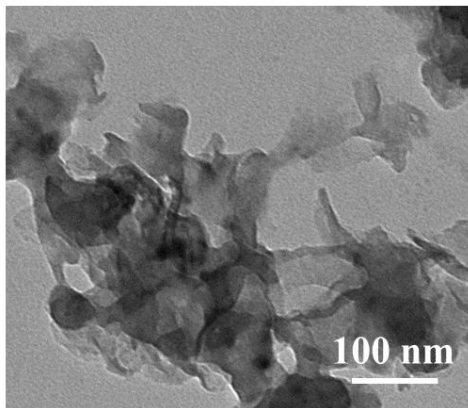

Figure S15. TEM images of the CFM-S ${ }_{30} \mathrm{NG}$ electrode after (a) 300 and (b) 2000 cycles. 

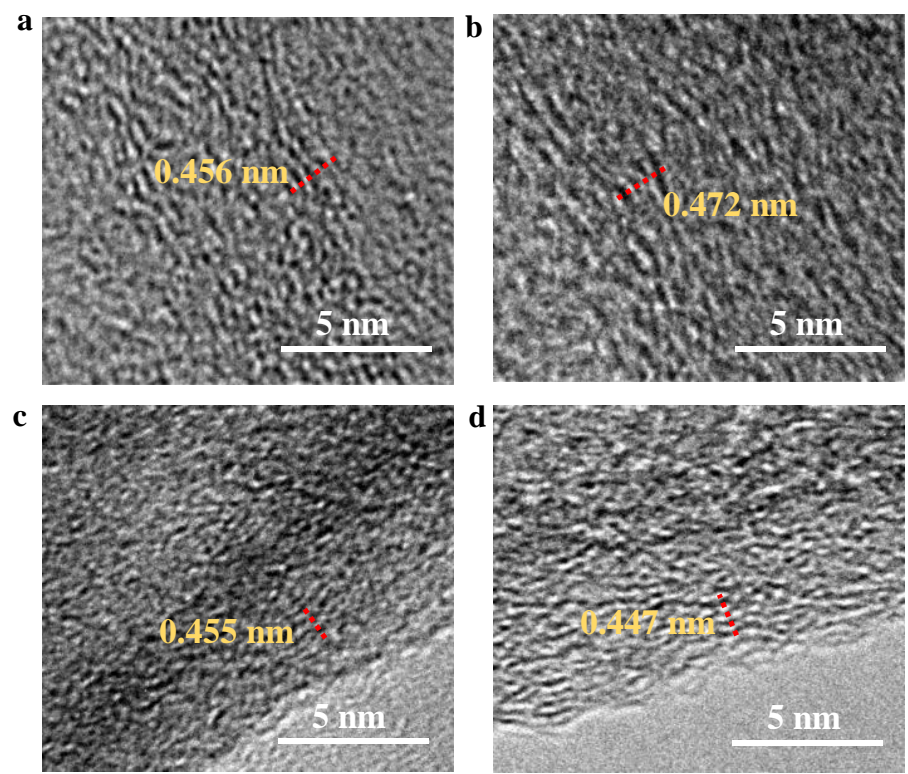

Figure S16 Ex-situ HRTEM images of the CFM-S ${ }_{30} \mathrm{NG}$ material with different potassiation states: (a) discharge to $1.0 \mathrm{~V}$, (b) discharge to $0.01 \mathrm{~V}$, (c) charge to $1.0 \mathrm{~V}$ and (d) charge to $2.5 \mathrm{~V}$. 

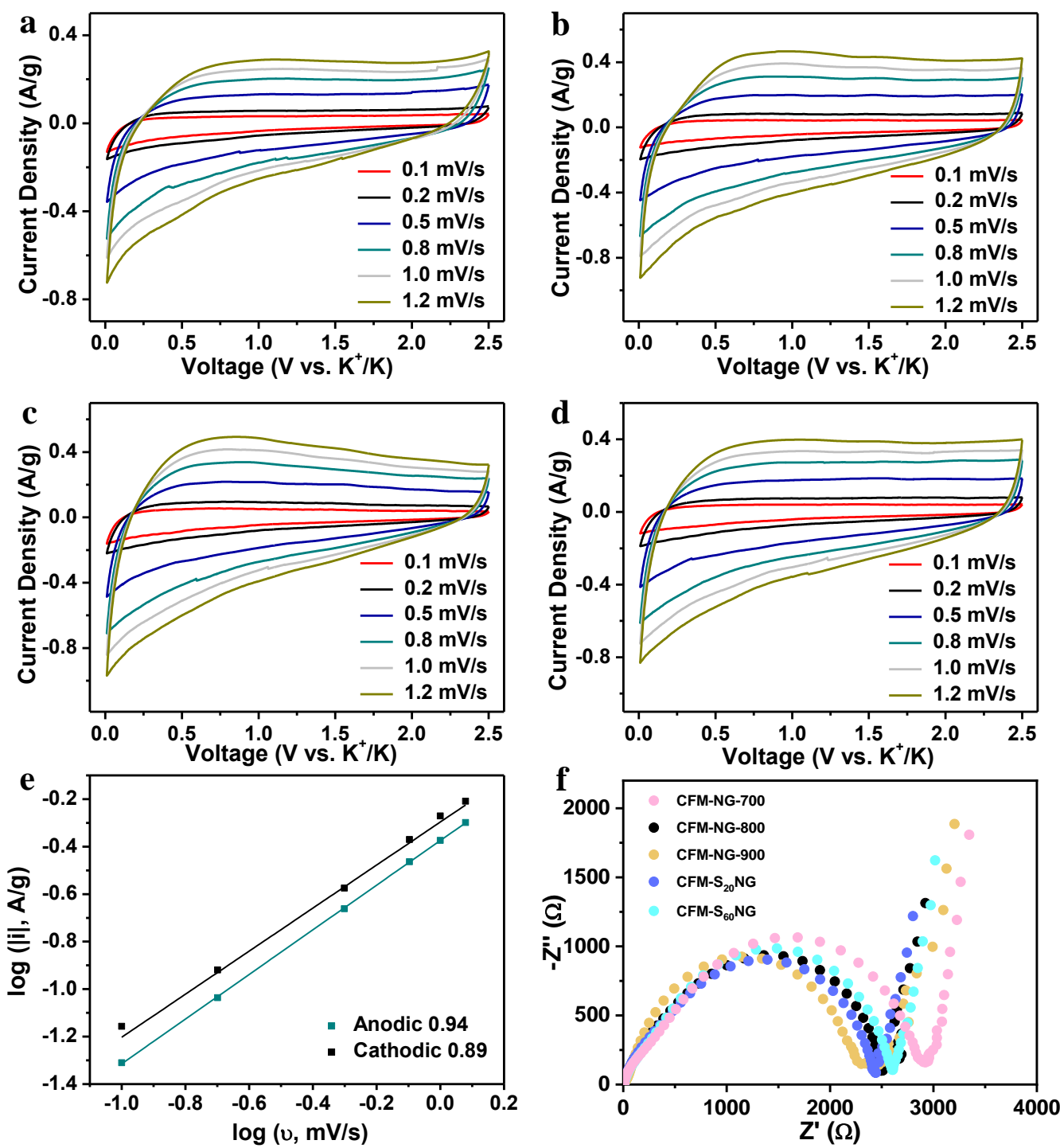

Figure S17 CV profiles of a series of NHCFs materials at different scan rates: (a) CFM-NG-700, (b) CFM-NG-800, (c) CFM-NG-900 and (d) CFM-S ${ }_{60}$ NG. (e) Determination of the b-value of CFM-S ${ }_{30} \mathrm{NG}$ using the relationship between peak current and scan rate in PIBs. (f) Nyquist curves of different materials. Herein, we assume the current (i) response obeys a power-law relationship $(\mathrm{i}=$ $\left.a v^{b}\right)$ with the scan rate (v), where the b-value can be determined by plotting the $\log (i) v s . \log (v)$ curves. Briefly, when the $b$-value is close to 1 , a surface capacitive process domitates, while $b$-value of 0.5 reveals an ideal Faradaic intercalation process. ${ }^{6}$ 
Table S1 Characteristics (XPS and BET surface area) of the different CFM-NG-T and CFM-S ${ }_{30} \mathrm{NG}$ materials.

\begin{tabular}{|c|c|c|c|c|c|}
\hline Materials & $\begin{array}{c}\mathrm{C} \\
(\text { at. \%) }\end{array}$ & $\begin{array}{c}\mathrm{N} \\
\text { (at. \%) }\end{array}$ & $\begin{array}{c}\text { O } \\
(\text { at. \%) }\end{array}$ & $\begin{array}{c}\mathrm{S} \\
(\text { at. \%) }\end{array}$ & $\begin{array}{c}\text { BET } \\
\text { surface } \\
\text { area }\left(\mathbf{m}^{2}\right. \\
\left.\mathbf{g}^{-1}\right)\end{array}$ \\
\hline CFM-NG-700 & 67.09 & 28.8 & 4.11 & & 214 \\
\hline CFM-NG-800 & 73.29 & 21.26 & 5.45 & & 261 \\
\hline CFM-NG-900 & 85.58 & 10.66 & 3.76 & & 440 \\
\hline CFM-S ${ }_{30} \mathrm{NG}$ & 74.47 & 18.79 & 5.56 & 1.19 & 296 \\
\hline
\end{tabular}

Table S2 The parameters calculated by the DFT method based on different active sites.

\begin{tabular}{cccccc}
\hline Materials & Graphite & N5 & N6 & S6 & N5+N6+S6 \\
\hline $\begin{array}{c}\text { Interlayer-distance } \\
(\AA)\end{array}$ & 3.167 & 3.188 & 3.180 & 3.187 & 3.193 \\
$\begin{array}{c}\text { Volume without K } \\
\left(\AA^{\wedge} 3\right)\end{array}$ & 1198.90 & 1202.04 & 1202.20 & 1209.67 & 1205.81 \\
$\begin{array}{c}\text { Volume with K } \\
\left(\AA^{\wedge} 3\right)\end{array}$ & 1379.35 & 1250.83 & 1203.15 & 1212.18 & 1222.09 \\
\hline $\begin{array}{c}\text { Volume expansion }(\%) \\
\text { Relative adsorption } \\
\text { energy }\left(\Delta \mathbf{E}_{\mathbf{a},}, \mathbf{e V}\right)\end{array}$ & 15.05 & 4.06 & 0.08 & 0.21 & 1.34 \\
\hline
\end{tabular}


Table S3. Comparison of PIBs performance of the CFM-S ${ }_{30} \mathrm{NG}$ with those reported anode materials.

\begin{tabular}{|c|c|c|c|}
\hline Materials & $\begin{array}{c}\text { Reversible Capacity \& } \\
\text { Rate Capability }\end{array}$ & $\begin{array}{c}\text { Cycle life } \\
\text { (capacity @ cycle } \\
\text { number@mA/g) }\end{array}$ & References \\
\hline $\mathrm{CFM} \mathrm{S}_{30} \mathrm{NG}$ & $\begin{array}{l}348.2 \mathrm{mAh} / \mathrm{g} @ 50 \mathrm{~mA} / \mathrm{g} \\
204.3 \mathrm{mAh} / \mathrm{g} @ 2000 \mathrm{~mA} / \mathrm{g}\end{array}$ & $\begin{array}{l}203.9 \mathrm{mAh} / \mathrm{g} @ 500 \text { cycles } \\
\text { @1000 mA/g } \\
202.1 \mathrm{mAh} / \mathrm{g} @ 1000 \text { cycles } \\
\text { @1000 mA/g } \\
188.8 \mathrm{mAh} / \mathrm{g} @ 2000 \text { cycles } \\
@ 1000 \mathrm{~mA} / \mathrm{g}\end{array}$ & This work \\
\hline $\begin{array}{c}\text { Reduced graphene } \\
\text { oxide }\end{array}$ & $\begin{array}{l}200 \text { mAh/g@ } 5 \text { mA/g } \\
50 \text { mAh/g@100mA/g }\end{array}$ & $\begin{array}{l}150 \text { mAh /g @ } 175 \text { cycles } \\
@ 10 \mathrm{~mA} / \mathrm{g}\end{array}$ & 7 \\
\hline $\begin{array}{c}\text { Graphitic carbon } \\
\text { nanocage }\end{array}$ & $\begin{array}{l}221 \mathrm{mAh} / \mathrm{g} @ 27.9 \mathrm{~mA} / \mathrm{g} \\
175 \mathrm{mAh} / \mathrm{g} @ 9765 \mathrm{~mA} / \mathrm{g}\end{array}$ & $\begin{array}{l}195 \text { mAh/g @ } 100 \text { cycles } \\
@ 56 \text { mA/g }\end{array}$ & 8 \\
\hline $\begin{array}{c}\text { Hard-soft composite } \\
\text { carbon }\end{array}$ & $\begin{array}{l}230 \mathrm{mAh} / \mathrm{g} \text { at } 140 \mathrm{~mA} / \mathrm{g} \\
167 \mathrm{mAh} / \mathrm{g} \text { at } 1400 \mathrm{~mA} / \mathrm{g}\end{array}$ & $\begin{array}{l}200 \text { mAh/g @ } 200 \text { cycles } \\
\text { @ } 280 \mathrm{~mA} / \mathrm{g}\end{array}$ & 9 \\
\hline $\begin{array}{c}\text { N- and O-rich carbon } \\
\text { nanofiber }\end{array}$ & $\begin{array}{l}230 \mathrm{mAh} / \mathrm{g} \text { at } 140 \mathrm{~mA} / \mathrm{g} \\
110 \mathrm{mAh} / \mathrm{g} \text { at } 2800 \mathrm{~mA} / \mathrm{g}\end{array}$ & $\begin{array}{l}170 \mathrm{mAh} / \mathrm{g} @ 1900 \mathrm{cycles} \\
\text { @ } 280 \mathrm{~mA} / \mathrm{g}\end{array}$ & 10 \\
\hline N-rich hard carbon & $\begin{array}{l}250 \mathrm{mAh} / \mathrm{g} \text { at } 33.6 \mathrm{~mA} / \mathrm{g} \\
156 \mathrm{mAh} / \mathrm{g} \text { at } 5040 \mathrm{~mA} / \mathrm{g}\end{array}$ & $\begin{array}{l}154 \text { mAh/g @ } 4000 \text { cycles } \\
\text { @ } 20160 \text { mA/g }\end{array}$ & 11 \\
\hline $\begin{array}{l}\text { P-doped N-rich } \\
\text { honeycomb-like } \\
\text { carbon }\end{array}$ & $\begin{array}{l}419.3 \text { mAh/g@100 mA/g } \\
268.1 \mathrm{mAh} / \mathrm{g} @ 1000 \mathrm{~mA} / \mathrm{g}\end{array}$ & $\begin{array}{l}270.4 \text { mAh/g@ } 1000 \text { cycles } \\
\text { @ } 1 \text { A/g }\end{array}$ & 12 \\
\hline $\begin{array}{l}\text { N/O dual-doped hard } \\
\text { carbon }\end{array}$ & $\begin{array}{l}304.6 \mathrm{mAh} / \mathrm{g} @ 100 \mathrm{~mA} / \mathrm{g} \\
178.9 \mathrm{mAh} / \mathrm{g} @ 5000 \mathrm{~mA} / \mathrm{g}\end{array}$ & $\begin{array}{l}189.5 \text { mAh/g@ } 5000 \text { cycles } \\
\text { @ } 1 \mathrm{~A} / \mathrm{g}\end{array}$ & 13 \\
\hline $\begin{array}{l}\text { Carbon quantum dot } \\
\text { micelles tailored } \\
\text { hollow carbon }\end{array}$ & $\begin{array}{l}254 \text { mAh/g @ 0.1 A/g } \\
145 \text { mAh/g @ } 4 \text { A/g }\end{array}$ & $\begin{array}{l}160 \text { mAh/g@ } 800 \text { cycles } \\
@ 1 \text { A/g }\end{array}$ & 14 \\
\hline
\end{tabular}




\begin{tabular}{|c|c|c|c|}
\hline $\begin{array}{l}\text { N/O co-doped } \\
\text { mesoporous carbon } \\
\text { octahedrons }\end{array}$ & $\begin{array}{l}354 \mathrm{mAh} / \mathrm{g} @ 50 \mathrm{~mA} / \mathrm{g} \\
110 \mathrm{mAh} / \mathrm{g} @ 1000 \mathrm{~mA} / \mathrm{g}\end{array}$ & $\begin{array}{l}100 \text { mAh/g @ } 1300 \text { cycles } \\
@ 1 \text { A/g }\end{array}$ & 15 \\
\hline $\begin{array}{l}\text { Hierarchically porous } \\
\text { N-doped carbon } \\
\text { fibers }\end{array}$ & $\begin{array}{l}197 \text { mAh/g@ } 50 \text { mA/g } \\
57 \text { mAh/g@ } @ 50 \text { mA/g }\end{array}$ & $\begin{array}{l}65 \text { mAh/g @ } 346 \text { cycles } \\
\text { @ } 100 \text { mA/g }\end{array}$ & 16 \\
\hline $\begin{array}{c}\text { Hierarchical carbon } \\
\text { nanotube }\end{array}$ & $\begin{array}{l}232 \mathrm{mAh} / \mathrm{g} @ 100 \mathrm{~mA} / \mathrm{g} \\
162 \mathrm{mAh} / \mathrm{g} @ 1600 \mathrm{~mA} / \mathrm{g}\end{array}$ & $\begin{array}{l}210 \text { mAh/g @ } 500 \text { cycles } \\
@ 100 \mathrm{~mA} / \mathrm{g}\end{array}$ & 17 \\
\hline $\begin{array}{c}\text { Carbon nanofiber } \\
\text { foam }\end{array}$ & $\begin{array}{l}240 \mathrm{mAh} / \mathrm{g} @ 50 \mathrm{~mA} / \mathrm{g} \\
164 \mathrm{mAh} / \mathrm{g} @ 1000 \mathrm{~mA} / \mathrm{g}\end{array}$ & $\begin{array}{l}158 \text { mAh/g @ } 2000 \text { cycles } \\
\text { @ } 1000 \text { mA/g }\end{array}$ & 18 \\
\hline $\begin{array}{l}\text { Sulphur-doped } \\
\text { reduced graphene } \\
\text { oxide }\end{array}$ & $\begin{array}{l}435 \text { mAh/g@ } 050 \text { mA/g } \\
224 \text { mAh/g@1 A/g }\end{array}$ & $\begin{array}{l}229 \text { mAh/g @ } 500 \text { cycles } \\
@ 1 \mathrm{~A} / \mathrm{g}\end{array}$ & 19 \\
\hline $\begin{array}{l}\text { N-doped few-layer } \\
\text { graphene }\end{array}$ & $\begin{array}{l}350 \mathrm{mAh} / \mathrm{g} @ 50 \mathrm{~mA} / \mathrm{g} \\
200 \mathrm{mAh} / \mathrm{g} @ 100 \mathrm{~mA} / \mathrm{g}\end{array}$ & $\begin{array}{l}210 \text { mAh/g@ } 100 \text { cycles } \\
@ 100 \mathrm{~mA} / \mathrm{g}\end{array}$ & 20 \\
\hline $\begin{array}{c}\text { S/O-codoped porous } \\
\text { hard carbon } \\
\text { microspheres }\end{array}$ & $\begin{array}{l}226.6 \mathrm{mAh} / \mathrm{g} @ 50 \mathrm{~mA} / \mathrm{g} \\
158 \mathrm{mAh} / \mathrm{g} @ 1000 \mathrm{~mA} / \mathrm{g}\end{array}$ & $\begin{array}{l}108.4 \text { mAh/g @ } 2000 \text { cycles } \\
\text { @ } 1000 \mathrm{~mA} / \mathrm{g}\end{array}$ & 21 \\
\hline $\begin{array}{c}\text { Hollow } \\
\text { interconnected } \\
\text { neuron-like carbon } \\
\text { architecture }\end{array}$ & $\begin{array}{l}340 \mathrm{mAh} / \mathrm{g} @ 28 \mathrm{~mA} / \mathrm{g} \\
\sim 120 \mathrm{mAh} / \mathrm{g} @ 560 \mathrm{~mA} / \mathrm{g}\end{array}$ & $\begin{array}{l}\sim 150 \text { mAh/g @ } 500 \text { cycles } \\
\text { @ } 280 \mathrm{~mA} / \mathrm{g}\end{array}$ & 22 \\
\hline $\begin{array}{l}\text { N/O-codoped } \\
\text { hierarchical porous } \\
\text { hard carbon }\end{array}$ & $\begin{array}{l}365 \mathrm{mAh} / \mathrm{g} @ 25 \mathrm{~mA} / \mathrm{g} \\
118 \mathrm{mAh} / \mathrm{g} @ 3000 \mathrm{~mA} / \mathrm{g}\end{array}$ & $\begin{array}{l}230.6 \text { mAh/g @ } 100 \text { cycles } \\
\text { @ } 50 \text { mA/g }\end{array}$ & 23 \\
\hline $\begin{array}{l}\text { N-doped carbon } \\
\text { nanofibers }\end{array}$ & $\begin{array}{l}238 \text { mAh/g@ } 100 \text { mA/g } \\
126 \text { mAh/g@ } 05000 \text { mA/g }\end{array}$ & $\begin{array}{l}248 \text { mAh/g @ } 100 \text { cycles } \\
@ 25 \mathrm{~mA} / \mathrm{g} \\
146 \mathrm{mAh} / \mathrm{g} @ 4000 \text { cycles } \\
@ 2 \mathrm{~A} / \mathrm{g}\end{array}$ & 24 \\
\hline
\end{tabular}




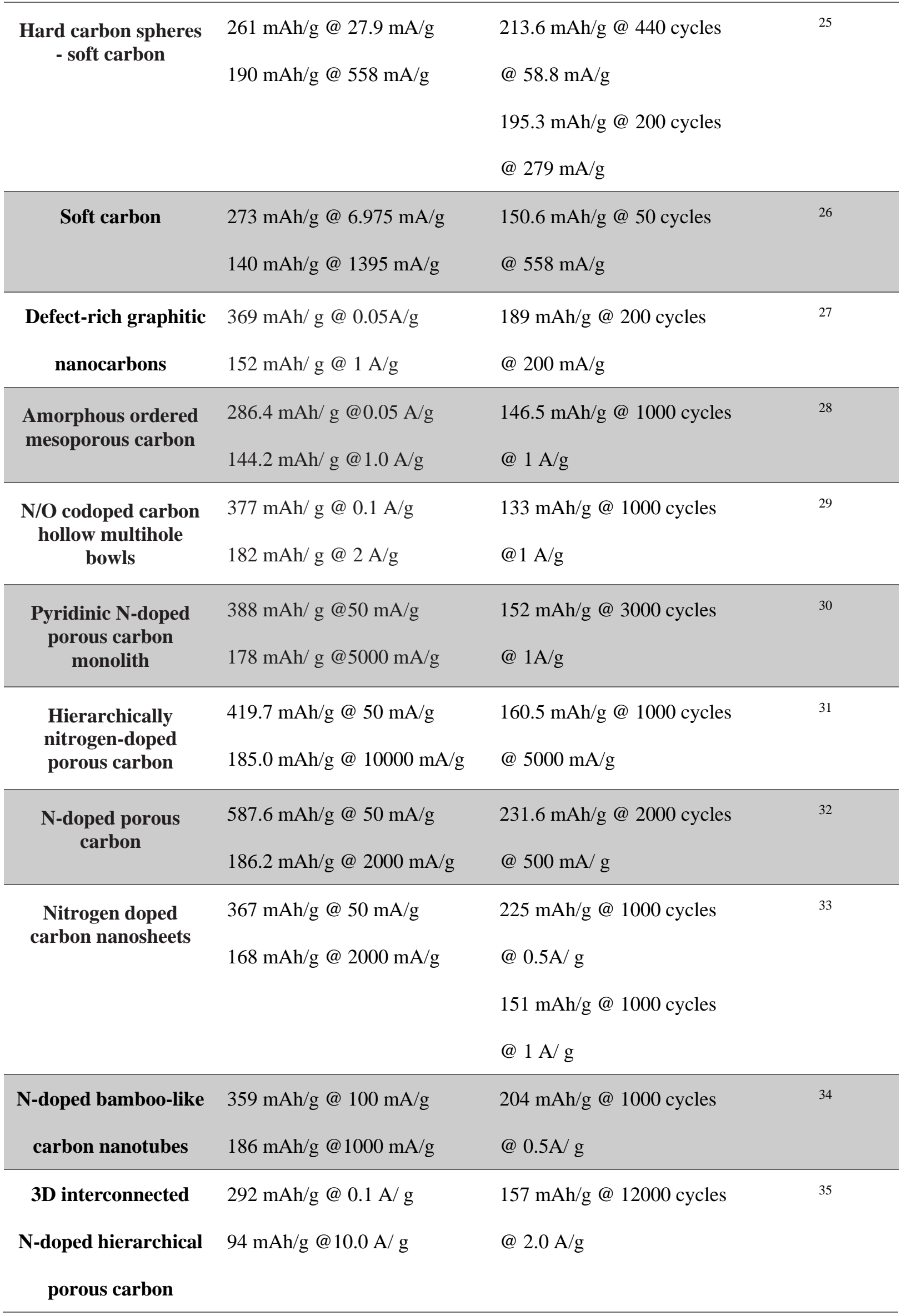


ultra-high

293.5 mAh/g @ 100 mA/g

161.3 mAh/g@ 1600 cycles

36

pyrrolic/pyridinic-N- $224 \mathrm{mAh} / \mathrm{g} @ 1000 \mathrm{~mA} / \mathrm{g} \quad$ @ $1000 \mathrm{~mA} / \mathrm{g}$

doped necklace-like

hollow carbon film 


\section{REFERENCES}

(1) Kresse, G.; Furthmuller, J., Efficient Iterative Schemes for Ab Initio Total-Energy Calculations Using a Plane-Wave Basis Set. Phys. Rev. B 1996, 54 (16), 11169-11186.

(2) Blochl, P. E., Projector augmented-wave method. Phys. Rev. B 1994, 50 (24), 17953-17979.

(3) Perdew, J. P.; Burke, K.; Ernzerhof, M., Generalized Gradient Approximation Made Simple. Phys. Rev. Lett. 1996, 77 (18), 3865-3868.

(4) Mills, G.; Jonsson, H.; Schenter, G. K., Reversible Work Transition-State Theory-Application to Dissociative Adsorption of Hydrogen. Surf. Sci. 1995, 324 (2-3), 305-337.

(5) Grimme, S., Semiempirical GGA-type Density Functional Constructed with a Long-Range Dispersion Correction. J. Comput. Chem. 2006, 27 (15), 1787-1799.

(6) Liu, Y.; Dai, H.; Wu, L.; Zhou, W.; He, L.; Wang, W.; Yan, W.; Huang, Q.; Fu, L.; Wu, Y., A Large Scalable and Low-Cost Sulfur/Nitrogen Dual-Doped Hard Carbon as the Negative Electrode Material for High-Performance Potassium-Ion Batteries. Adv. Energy Mater.2019, 9 (34), 1901379.

(7) Luo, W.; Wan, J.; Ozdemir, B.; Bao, W.; Chen, Y.; Dai, J.; Lin, H.; Xu, Y.; Gu, F.; Barone, V.; $\mathrm{Hu}$, L., Potassium Ion Batteries with Graphitic Materials. Nano Lett. 2015, 15 (11), 7671-7677.

(8) Cao, B.; Zhang, Q.; Liu, H.; Xu, B.; Zhang, S.; Zhou, T.; Mao, J.; Pang, W. K.; Guo, Z.; Li, A.; Zhou, J.; Chen, X.; Song, H., Graphitic Carbon Nanocage as a Stable and High Power Anode for Potassium-Ion Batteries. Adv. Energy Mater. 2018, 8 (25), 1801149.

(9) Jian, Z. L.; Hwang, S.; Li, Z. F.; Hernandez, A. S.; Wang, X. F.; Xing, Z. Y.; Su, D.; Ji, X. L., Hard-Soft Composite Carbon as a Long-Cycling and High-Rate Anode for Potassium-Ion Batteries. Adv. Funct. Mater. 2017, 27 (26), 1700324.

(10) Adams, R. A.; Syu, J.-M.; Zhao, Y.; Lo, C.-T.; Varma, A.; Pol, V. G., Binder-Free N- and O-Rich Carbon Nanofiber Anodes for Long Cycle Life K-Ion Batteries. ACS Appl. Mater. Inter. 2017, 9 (21), 17872-17881.

(11) Chen, C.; Wang, Z.; Zhang, B.; Miao, L.; Cai, J.; Peng, L.; Huang, Y.; Jiang, J.; Huang, Y.; Zhang, L.; Xie, J., Nitrogen-Rich Hard Carbon as a Highly Durable Anode for High-Power Potassium-Ion Batteries. Energy Storage Mater. 2017, 8, 161-168.

(12) He, H.; Huang, D.; Tang, Y.; Wang, Q.; Ji, X.; Wang, H.; Guo, Z., Tuning Nitrogen Species in Three-Dimensional Porous Carbon via Phosphorus Doping for Ultra-Fast Potassium Storage. Nano Energy 2019, 57, 728-736.

(13) Cui, R. C.; Xu, B.; Dong, H. J.; Yang, C. C.; Jiang, Q., N/O Dual-Doped Environment-Friendly Hard Carbon as Advanced Anode for Potassium-Ion Batteries. Adv. Sci. 2020, 1902547.

(14) Hong, W.; Zhang, Y.; Yang, L.; Tian, Y.; Ge, P.; Hu, J.; Wei, W.; Zou, G.; Hou, H.; Ji, X., Carbon Quantum Dot Micelles Tailored Hollow Carbon Anode for Fast Potassium And Sodium Storage. Nano Energy 2019, 65, 104038.

(15) Xia, G.; Wang, C.; Jiang, P.; Lu, J.; Diao, J.; Chen, Q., Nitrogen/Oxygen Co-Doped Mesoporous Carbon Octahedrons for High-Performance Potassium-Ion Batteries. J. Mater. Chem. A 2019, 7 (19), 12317-12324.

(16) Zhang, M.; Shoaib, M.; Fei, H.; Wang, T.; Zhong, J.; Fan, L.; Wang, L.; Luo, H.; Tan, S.; Wang, Y.; Zhu, J.; Hu, J.; Lu, B., Hierarchically Porous N Doped Carbon Fibers as a Free-Standing 
Anode for High-Capacity Potassium-Based Dual-Ion Battery. Adv. Energy Mater. 2019, 1901663.

(17) Wang, Y.; Wang, Z.; Chen, Y.; Zhang, H.; Yousaf, M.; Wu, H.; Zou, M.; Cao, A.; Han, R. P. S., Hyperporous Sponge Interconnected by Hierarchical Carbon Nanotubes as a High-Performance Potassium-Ion Battery Anode. Adv. Mater. 2018, 30 (32), 1802074.

(18) Li, H.; Cheng, Z.; Zhang, Q.; Natan, A.; Yang, Y.; Cao, D.; Zhu, H., Bacterial-Derived, Compressible, and Hierarchical Porous Carbon for High-Performance Potassium-Ion Batteries. Nano Lett. 2018, 18 (11), 7407-7413.

(19) Li, J.; Qin, W.; Xie, J.; Lei, H.; Zhu, Y.; Huang, W.; Xu, X.; Zhao, Z.; Mai, W., Sulphur-Doped Reduced Graphene Oxide Sponges as High-Performance Free-Standing Anodes for K-Ion Storage. Nano Energy 2018, 53, 415-424.

(20) Share, K.; Cohn, A. P.; Carter, R.; Rogers, B.; Pint, C. L., Role of Nitrogen-Doped Graphene for Improved High-Capacity Potassium Ion Battery Anodes. ACS Nano 2016, 10 (10), 9738-9744.

(21) Chen, M.; Wang, W.; Liang, X.; Gong, S.; Liu, J.; Wang, Q.; Guo, S.; Yang, H., Sulfur/Oxygen Codoped Porous Hard Carbon Microspheres for High-Performance Potassium-Ion Batteries. Adv. Energy Mater. 2018, 8 (19), 1800171.

(22) Bin, D.-S.; Lin, X.-J.; Sun, Y.-G.; Xu, Y.-S.; Zhang, K.; Cao, A.-M.; Wan, L.-J., Engineering Hollow Carbon Architecture for High-Performance K-Ion Battery Anode. J. Am. Chem. Soc. 2018, 140 (23), 7127-7134.

(23) Yang, J.; Ju, Z.; Jiang, Y.; Xing, Z.; Xi, B.; Feng, J.; Xiong, S., Enhanced Capacity and Rate Capability of Nitrogen/Oxygen Dual-Doped Hard Carbon in Capacitive Potassium-Ion Storage. Adv. Mater. 2018, 30 (4), 1700104.

(24) Xu, Y.; Zhang, C.; Zhou, M.; Fu, Q.; Zhao, C.; Wu, M.; Lei, Y., Highly Nitrogen Doped Carbon Nanofibers with Superior Rate Capability and Cyclability for Potassium Ion Batteries. Nat. Commun. 2018, 9, 1720.

(25) Jian, Z.; Hwang, S.; Li, Z.; Hernandez, A. S.; Wang, X.; Xing, Z.; Su, D.; Ji, X., Hard-Soft Composite Carbon as a Long-Cycling and High-Rate Anode for Potassium-Ion Batteries. Adv. Funct. Mater. 2017, 27 (26), 1700324.

(26) Jian, Z.; Luo, W.; Ji, X., Carbon Electrodes for K-ion Batteries. J. Am. Chem. Soc. 2015, 137 (36), 11566-11569.

(27) Zhang, W.; Ming, J.; Zhao, W.; Dong, X.; Hedhili, M. N.; Costa, P. M. F. J.; Alshareef, H. N., Graphitic Nanocarbon with Engineered Defects for High-Performance Potassium-Ion Battery Anodes. Adv. Funct. Mater. 2019, 29 (35), 1903641.

(28) Wang, W.; Zhou, J.; Wang, Z.; Zhao, L.; Li, P.; Yang, Y.; Yang, C.; Huang, H.; Guo, S., Short-Range Order in Mesoporous Carbon Boosts Potassium-Ion Battery Performance. Adv. Energy Mater. 2018, 8 (5), 1701648.

(29) Zhang, Z.; Jia, B.; Liu, L.; Zhao, Y.; Wu, H.; Qin, M.; Han, K.; Wang, W. A.; Xi, K.; Zhang, L.; Qi, G.; Qu, X.; Kumar, R. V., Hollow Multihole Carbon Bowls: a Stress-Release Structure Design for High-Stability and High-Volumetric-Capacity Potassium-Ion Batteries. ACS Nano 2019, 13 (10), 11363-11371. 
(30) Xie, Y.; Chen, Y.; Liu, L.; Tao, P.; Fan, M.; Xu, N.; Shen, X.; Yan, C., Ultra-High Pyridinic N-Doped Porous Carbon Monolith Enabling High-Capacity K-Ion Battery Anodes for both Half-cell and Full-cell Applications. Adv. Mater. 2017, 29 (35), 1702268.

(31) Li, D.; Ren, X.; Ai, Q.; Sun, Q.; Zhu, L.; Liu, Y.; Liang, Z.; Peng, R.; Si, P.; Lou, J.; Feng, J.; Ci, L., Facile Fabrication of Nitrogen-Doped Porous Carbon as Superior Anode Material for Potassium-Ion Batteries. Adv. Energy Mater. 2018, 1802386.

(32) Li, Y.; Yang, C.; Zheng, F.; Ou, X.; Pan, Q.; Liu, Y.; Wang, G., High Pyridine N-Doped Porous Carbon Derived from Metal-Organic Frameworks for Boosting Potassium-Ion Storage. J. Mater. Chem. A 2018, 6 (37), 17959-17966.

(33) Liu, L.; Chen, Y.; Xie, Y.; Tao, P.; Li, Q.; Yan, C., Understanding of the Ultrastable K-Ion Storage of Carbonaceous Anode. Adv. Funct. Mater. 2018, 28 (29), 1801989.

(34) Liu, Y.; Yang, C.; Pan, Q.; Li, Y.; Wang, G.; Ou, X.; Zheng, F.; Xiong, X.; Liu, M.; Zhang, Q., Nitrogen-Doped Bamboo-Like Carbon Nanotubes as Anode Material for High Performance Potassium Ion Batteries. J. Mater. Chem. A 2018, 6 (31), 15162-15169.

(35) Zhou, X.; Chen, L.; Zhang, W.; Wang, J.; Liu, Z.; Zeng, S.; Xu, R.; Wu, Y.; Ye, S.; Feng, Y.; Cheng, X.; Peng, Z.; Li, X.; Yu, Y., Three-Dimensional Ordered Macroporous Metal-Organic Framework Single Crystal-Derived Nitrogen-Doped Hierarchical Porous Carbon for High-Performance Potassium-Ion Batteries. Nano Lett. 2019, 19 (8), 4965-4973.

(36) Yang, W.; Zhou, J.; Wang, S.; Zhang, W.; Wang, Z.; Lv, F.; Wang, K.; Sun, Q.; Guo, S., Freestanding Film Made by Necklace-Like N-Doped Hollow Carbon with Hierarchical Pores for High-Performance Potassium-Ion Storage. Energ. Environ. Sci. 2019, 12 (5), 1605-1612. 